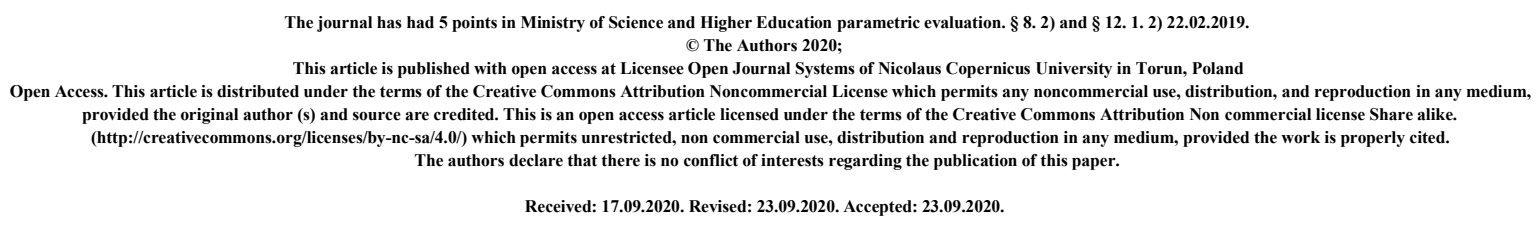

\title{
Adiponectin as novel biomarker of endothelial dysfunction in insulin resistance and obesity - a narrative review
}

\author{
Joanna Szydełko'a , Paulina Trojanowska ${ }^{1,2 b}$, Izabela Dąbrowska ${ }^{3 \mathrm{c}}$, \\ Magdalena Szydełko-Gorzkowicz ${ }^{4 d}$, Michał Litwińczuk ${ }^{5 e}$
}

${ }^{1}$ Department of Endocrinology, Medical University of Lublin, Poland

${ }^{2}$ Department of Pediatric Neurology, Medical University of Lublin, Poland

${ }^{3}$ Department of Interventional Radiology and Neuroradiology, Medical University of Lublin, Poland

${ }^{4}$ Independent Public Clinical Hospital No. 4 in Lublin, Poland

${ }^{5}$ Endocrinology Outpatient Clinic, Farmed Non-Public Healthcare Institution, Lublin, Poland

a jszydelko@interia.pl, ORCID ID: https://orcid.org/0000-0003-3744-9058

b trojanowska_paulina@wp.pl, ORCID: https://orcid.org/0000-0002-5289-6473

c nematoda@gmail.com, ORCID: https://orcid.org/0000-0003-1593-2284

d mszydelko@interia.pl, ORCID ID: https://orcid.org/0000-0001-6216-9934

emlitwinczuk405@gmail.com, ORCID ID: https://orcid.org/0000-0002-4086-6779

\section{Corresponding author:}

Joanna Szydełko

Department of Endocrinology

Jaczewskiego 8 Street

20-954 Lublin, Poland

phone: +48 817244668

e-mail: jszydelko@interia.pl 


\begin{abstract}
Introduction: Obesity is a chronic fatal disease with still growing incidence among children, adolescents, and adults worldwide. The subclinical inflammatory process together with hipoadiponectinemia may lead to the development of various comorbidities, including cardiovascular complications. That is why, the relationship between adipose tissue activity, obesity, insulin resistance, and endothelial function is in high interest and an object of extensively studies.
\end{abstract}

Aim of the study: This article summarizes the current knowledge on the anti-atherogenic effects of adiponectin and its properties to improve endothelial function in obesity-related insulin resistance.

Description of knowledge: Adiponectin, an adipose tissue-derived pleiotropic hormone with anti-inflammatory, anti-atherogenic, anti-diabetic, and insulin-sensitizing actions, is not only engaged in modulation of type 2 diabetes mellitus, hypertension or coronary artery disease, but the latest researches highlight its role in improving vascular wall integrity. It affects complex signaling pathways in endothelial cells and influence inflammatory responses in the subendothelial space. Pre-clinical and clinical studies suggest that agents leading to increase in adiponectin levels, simultaneously contribute to decrease insulin resistance, and improve endothelial dysfunction.

Conclusions: Adiponectin may be a predictive factor of endothelial dysfunctionality and vascular remodeling development in the group of patients with overweight, obesity, and insulin resistance. Discovering pharmacological agents and non-pharmacological interventions that increase the level of circulating adiponectin will become novel and innovative therapeutic strategy to ameliorate obesity-related comorbidities. Therefore, further studies are required to determine the exact role of adiponectin in the pathogenesis of metabolic diseases.

Key words: adiponectin; insulin resistance; obesity; vascular dysfunction 


\section{Introduction}

Obesity is a chronic disease characterized by the excessive deposition of fat that may lead to various comorbidities, including cardiovascular complications, diabetes mellitus, and cancers $[1,2]$. Overweight and obesity are commonly defined by the body mass index (BMI) calculated as weight ( $\mathrm{kg}$ ) divided by height (meters) squared. Individuals are classified to one of five categories according to BMI as normal weight: $18.5-24.9 \mathrm{~kg} / \mathrm{m}^{2}$, overweight: $25.0-29.9$ $\mathrm{kg} / \mathrm{m}^{2}$, grade I obesity: $30.0-34.9 \mathrm{~kg} / \mathrm{m}^{2}$, grade II obesity: $35.0-39.9 \mathrm{~kg} / \mathrm{m}^{2}$, and grade III obesity, also called severe or morbid obesity: $\geq 40 \mathrm{~kg} / \mathrm{m}^{2}[3,4]$. In the twenty-first century, obesity has reached global epidemic proportions with still increasing prevalence among children, adolescents, and adults. It is estimated that if current trends continue, about $58 \%$ of adults will be overweight or obese by 2030, that is why body weight disturbances are treated as major health, psychological, and socioeconomic problems worldwide $[1,5,6]$. What is more, date analysis of 900,000 adult patients from the Prospective Studies Collaboration (PSC) demonstrated that median survival rate may decrease by 8 to even 10 years for obese individuals with BMI of $40-45 \mathrm{~kg} / \mathrm{m}^{2}$, compared to those with normal BMI, mostly due to vascular complications [7]. Another simple and widely available measurement is waist circumference that allows to assess the distribution of adipose tissue. Currently, according to the values proposed by the International Diabetes Federation for European countries, waist circumference $\geq 80 \mathrm{~cm}$ in women and $\geq 94 \mathrm{~cm}$ in men are treated as threshold values for which abdominal obesity is diagnosed [8]. Moreover, waist-hip ratio (WHR) constitutes an important indicator for distinguishing the main types of obesity: gynoid obesity, slightly more typical for women, and visceral obesity, more characteristic in men. It is established by experts in many guidelines that WHR equal or higher than 1 for men and 0.8 for women indicates obesity of the abdominal type, and lower than these values suggests obesity of the gluteal-femoral type, although there are ethnic-specific WHR cutoffs in different studied populations [9,10]. Nevertheless, both BMI, waist circumference, and WHR do not provide accurate information about fat distribution, body composition, adipose tissue activity, and the related risk of cardiometabolic complications, morbidity, and mortality in obese individuals. Therefore, there is a great need to discover novel biomarkers for early predicting of endothelial or vascular dysfunction in insulin resistance, overweight, and obesity.

Since adipose tissue has been recognized as one of the largest metabolically active endocrine organ, but not only place for energy and lipid storage, it is an object of many 
researches in the context of glucose homeostasis and development of cardiovascular diseases $[11,12]$. Adipose tissue synthesizes and releases multiple inflammatory- and immunemodulatory proteins, such as cytokines, hormones, growth and vasoactive factors, and extracellular matrix proteins, collectively named as adipokines, that affect the functioning of the whole organism and are engaged in various physiological and pathophysiological processes [13,14]. Among all secreted molecules, adiponectin, also known as adipocyte complement-related protein of $30 \mathrm{kDa}$ (Acrp30), AdipoQ, apM1, and gelatin binding protein of $28 \mathrm{kD}$ (GBP-28), structurally belonging to $\mathrm{C} 1 \mathrm{q} / \mathrm{TNF}$ protein family, is one of the most extensively investigated novel cytokine and promising therapeutic target in obesity-related diseases $[15,16]$. The results of recent years' studies have revealed the link between anti- and proinflammatory adipokines' imbalance in obesity-induced insulin resistance in adipose tissue, and endothelial dysfunction, vascular remodelling as well as have demonstrated the novel pathways involved in the pathogenesis of atherosclerosis development [17-19]. What is interesting, but not widely known, chronic insulin resistance state has been strengthened by epidemiological and experimental studies as independent factor increasing the risk of cardiovascular disorders [20]. A thorough understanding of above-mentioned correlations and disturbances in adipose tissue microenvironment will likely result in innovative approach to diagnostic process and rationalizing treatment methods of these highly prevalent disorders in the future.

\section{Aim of the study}

The aim of this systematic review was to present the protective influence of adiponectin on endothelial and vascular function in the course of insulin resistance and obesity. Moreover, we also discussed the current and future perspectives of the usefulness of this adipocytokine as diagnostic and prognostic factor as well as potential therapeutic target in obesity-related vascular damages.

\section{Materials and methods}

The available literature was subjectively selected due to its usefulness in showing clinical approach to the role of adiponectin, an insulin-sensitizing and anti-inflammatory adipocytokine, as a biomarker of endothelial dysfunction in overweight and obesity. Furthermore, its involvement in the mechanisms of insulin resistance and atherosclerosis development was evaluated. Data which reveals inconsistency in results was shown as well. Articles in English in the EBSCO and the PubMed database have been analyzed using key words in various combinations: 'adiponectin', 'endothelial dysfunction', 'insulin resistance', 'overweight', 'obesity', 'metabolic disorders', 'diagnostic marker' and 'therapeutic target'. 


\section{Adiponectin, obesity and insulin resistance - when the vicious circle begins}

Obesity is a complex condition with a multifactorial background, including genetic, epigenetic, behavioral, socioeconomic, and environmental factors, in which a prolonged status of positive energy balance leads to both adipocyte hypertrophy and hyperplasia [21]. In consequence, the changes in size and number of adipocyte cells with further increase in the total fat mass, cause adipose tissue dysfunctionality and initiate a variety of inflammatory and immune signaling pathways that strongly contribute to disruption of metabolic homeostasis in the whole human organism [22]. Numerous in vitro and in vivo studies proved that adipose tissue in overnutrition conditions undergoes different cellular and structural reconstruction processes involving impaired angiogenesis leading to local hypoxia, mitochondrial dysfunction, changes in membrane proteins, increased adipocyte cell death due to apoptosis/necrosis and autophagy, disturbed extracellular matrix remodelling, and fibrosis [13,23-25]. It is also worth mentioning that adipose tissue expansion is strongly associated with innate and adaptive immune disturbances, such as increased infiltration of activated macrophages, neutrophils, foam cells, proinflammatory Th1 and Th17 CD4, B cells, mastocytes, and dendritic cells [26]. Above-mentioned disorders occur already at the early stages of obesity development, and then they are sustained by chronic low-grade inflammation, and at the same time exaggerate it. What is more, excessive visceral fat accumulation promotes unbalanced secretion of proinflammatory molecules, such as interleukin-6 (IL-6), interleukin-1 $\beta$ (IL-1 $)$, tumor necrosis factor-alpha (TNF- $\alpha$ ), leptin, monocyte chemoattractant protein 1 (MCP-1), which together with increased production of Creactive protein (CRP) by hepatocytes, subsequently inhibits adiponectin expression on both mRNA and protein levels, and thereby cause adipose tissue inflamed [27].

Adiponectin, a 244 amino acids protein encoded by the APM1 gene located on the long arm of the third chromosome, is one of the key adipose tissue-derived substances with a pleiotropic influence on glucose homeostasis, insulin sensitivity, lipid metabolism, and vascular endothelium integrity [15,28]. It is predominantly produced by mature cells of white adipose tissue with special attention to visceral fat compartments associated with the highest cardiovascular risk, subcutaneous, perivascular, and epicardial fat depots, although it may also be secreted by cardiomyocytes, hepatocytes, skeletal muscle, colon, salivary gland, placenta, and even hypophysis at significantly lower concentrations [28]. Adiponectin acts not only locally in adipose tissue in an autocrine and paracrine manner, but it also circulates in the bloodstream in three major multimeric forms, which include low molecular weight (LMW) trimers, middle molecular weight (MMW) hexamers, and high molecular weight (HMW) 
multimers, which is suggested to be the most active form, and thus it regulates processes in other tissues and distant organs [29]. It comprises about $0.01-0.05 \%$ of all plasma proteins and its level ranges from 3 to $30 \mu \mathrm{g} / \mathrm{ml}$ with about $40 \%$ higher concentration in women than in men (median: $8.7 \mu \mathrm{g} / \mathrm{ml}$ vs $5.5 \mu \mathrm{g} / \mathrm{ml}$ ) [28,30-32]. This highly bioactive molecule (a half-life of $75 \mathrm{~min}$ to $150 \mathrm{~min}$ ) with anti-inflammatory, anti-atherogenic, anti-diabetic, and insulinsensitizing properties exerts plentiful impact on diverse disease areas via two main transmembrane receptors: AdipoR1, primarily expressed in skeletal muscle and activated by AMPK (AMP-dependent protein kinase) phosphorylation, and AdipoR2, mainly localized in liver and involved in the activation of peroxisome proliferator activating receptor alpha (PPAR- $\alpha$ ), whereas vascular effects are mediated throughout unique cell surface molecule in endothelial and smooth muscle cells called T-cadherin receptor, which is able to bind MMW and HMW, but not LMW adiponectin form [15,31].

Furthermore, adiponectin stimulates fatty acid oxidation, partly due to the activation of AMPK signaling axis and subsequent deactivation of acetyl coenzyme A carboxylase, and in this way it decreases the level of circulating free fatty acids and improves insulin sensitivity [15]. However, high fat diets lead to the reduction in insulin-stimulated glucose transport, which together with chronic subclinical inflammatory process, altered adipokine patterns play a central role in the promotion of insulin resistance that is observed in even $50-70 \%$ of overweight or obese individuals [33,34]. Some authors claimed adiponectin levels influence the fluctuations in insulin sensitivity in even $73 \%$ of cases [35]. According to the current knowledge, in obesity-induced insulin resistance, down-regulation of adiponectin and its receptors expression is observed, and it can induce adiponectin resistance consistently developing during the progression of obesity. Although, it is known that AdipoR2 expression within visceral adipose tissue is decreased without any changes in AdipoR1 expression. It is also worth nothing that adaptor protein-containing pleckstrin homology domain, phosphotyrosine-binding domain, and leucine zipper motif 1 (APPL1), the intracellular binding partner of AdipoR1 and AdipoR2 complex and the mediator of adiponectin dependent insulin sensitization, may be a possible link between adiponectin-insulin resistance cross-talk, which causes multiple intracellular signal transduction disorders in obese individuals [32].

A growing number of pre-clinical and clinical studies repeatedly demonstrated that plasma adiponectin levels are negatively correlated with BMI, plasma insulin concentrations, and insulin resistance calculated using the HOMA-IR (Homeostatic Model Assessment for Insulin Resistance) index [36-37]. At the same time, it is suggested that HMW adiponectin better predicts insulin resistance and the metabolic syndrome in patients than total adiponectin 
concentration [38]. Many authors have shown that hypoadiponectinemia is observed in human and animals subjects with overweight and obesity compared to non-obese ones, but adiponectin levels are further decreased in patients with coexisting type 2 diabetes mellitus or cardiovascular diseases [28,39]. Notwithstanding, there are single reports in the literature, which do not prove the changes in adiponectin levels in individuals regarded as obese [40].

The initial hypothesis indicating that obesity induces chronic subclinical inflammation, and recent insights into immune and biochemical alteration highlights the leading role of adiponectin in the development of obesity-linked comorbidities. On the other hand, it seems that complex pathological mechanisms underlying the relationship between overweight or obesity, insulin resistance, altered adipokine profiles, and endothelial complications, make it hard to establish the moment when this vicious circle begins.

\section{Adiponectin and endothelial dysfunction in obesity-induced insulin resistance}

According to the latest data, it was found that the substantially higher risk of vascular and endothelial dysfunction as well as adverse cardiovascular events in the group of patients with metabolically unhealthy obesity compared to those with metabolically healthy obesity and metabolically healthy people of a normal weight is associated with imbalanced secretion of adipocytokines, mainly adiponectin [41-44]. Extensive experimentations in both animal models and humans have demonstrated that chronic low-grade inflammation, disturbed nitric oxide (NO)-bioavailability, oxidized low-density lipoprotein (oxLDL), insulin resistance together with hypoadiponectinemia are involved in the initiation and progression of obesityinduced disruption of vascular homeostasis, endothelial vasodilator dysfunction, which result in the development of atherosclerosis, and then micro- and macrovascular complications [45]. What is interesting, the research conducted by Muñoz-Muñoz E et al. shed a new light on the pathogenesis of vascular dysfunction as they have revealed that placental endothelium of large-for-gestational-age (LGA) neonates from obese mothers is characterized with diminished vascular response to adiponectin compared to fetoplacental chorionic arteries and umbilical cord endothelium of adequate-for-gestational (AGA) newborns. Similar observations were also presented in primary cultures of umbilical artery endothelial cells of LGAs from mothers with pre-gestational obesity, in which decreased endothelial nitric oxide synthase (eNOS) activation and adiponectin-dependent NO production were proved. These molecular changes indicate that the onset of cardiovascular disorders is already present in the early period of human life, especially in genetically predisposed subjects with programmed obesity-related complications [46]. 
Adiponectin, a unique substance with anti-inflammatory, vasculoprotective, and angiogenic functions, is involved in the prevention of atherosclerotic process development at all of its steps by both affecting highly complex signaling pathways in endothelial cells and modulating inflammatory responses in the subendothelial space [18]. Primarily, it is engaged in down-regulation of endothelial adhesion molecules expression, such as vascular cell adhesion molecule-1 (VCAM-1), intracellular adhesion molecule-1 (ICAM-1), and E-selectin, which bind mononuclear cells, and in this way enable their migration into the subendothelial space and actively promote atherogenesis. Adiponectin also plays a role as an inhibitor of transformation of monocyte-derived macrophages into foam cells by inhibiting the class A macrophage scavender receptor, and it is suggested to prevent binding of LDL fraction to vascular proteoglycan. These actions aim to prevent the atherosclerotic plaque formation, and lead to decreased level of local inflammation. In addition, adiponectin protects against pathological vascular remodelling through suppression of smooth muscle cell growth and their migration. What is more, atheroprotective effects of adiponectin also include its impact on the vasculature by reducing platelet aggregation [27,45]. Besides, adiponectin improves endothelial vasorelaxation by increasing eNOS activity and NO production, and at the same time it suppresses the generation of reactive oxygen species (ROS) (superoxide and $\mathrm{H}_{2} \mathrm{O}_{2}$ ), which are released in response to several triggers, such as free fatty acids, oxLDL, TNF- $\alpha$ that activate adequate signaling kinases $[18,19]$. Moreover, this cytokine stimulates angiogenesis and recruitment of progenitor cells. These effects of adiponectin are mainly mediated through increased phosphorylation of the insulin receptor, activation of AMP-activated protein kinase, and modulation of the nuclear factor $\kappa \mathrm{B}$ pathway $[45,47]$.

The above-described properties of adiponectin were proved in both experimental and clinical studies [17,48-50]. Ortiz Segura MDC et al. indicated that the adiponectin concentrations were lower and soluble ICAM-1 levels as markers of endothelium dysfunction were increased in Mexican obese adolescents with insulin resistance compared to those without insulin resistance and in the controls [17]. The results of the research conducted on rat models revealed that the implementation of a high-fat diet leads to continuously increasing of pro-inflammatory cytokines, and decreasing of anti-inflammatory adiponectin levels with the first effects observed after six weeks, while NO levels were decreased at six weeks and lipid peroxidation increased in twelve weeks, which in consequence induced an increase in systolic blood pressure [48]. Similar observations were found in adiponectin-deficient mice which developed enhanced salt- or atherogenic diet-induced hypertension due to disturbances in the endothelial cell function $[49,50]$. Furthermore, it turned out that adiponectin administration 
may reverse neointimal thickening in mechanically injured arteries due to inhibitory effects of this cytokine on proliferation and migration of vascular smooth muscle cells [51]. On the other hand, there are several studies suggesting that no association between adiponectin levels and cardiovascular risk is observed as well as increased adiponectin concentrations are associated with a worse prognosis in patients with cardiometabolic disturbances, although the mechanisms of such paradox remain still unclear [52].

\section{Adiponectin as potential therapeutic target for obesity-related vascular damage}

Adiponectin due to its multiple properties seems to be not only relevant clinical biomarker, but also promising therapeutic target for obesity-related vascular and endothelial damages [53]. Currently, there are numerous studies that assess alteration in adiponectin concentrations after both non-pharmacological and pharmacological interventions, but many of them were conducted on animal models or in in vitro conditions. Clinical trials that would comprehensively evaluate efficacy of various treatment strategies contributing to increase in adiponectin levels and improving endothelial functions in group of patients with overweight, obesity, and insulin resistance, especially before prediabetes state, type 2 diabetes mellitus or cardiovascular complications develop, are limited [54].

Among the common non-pharmacological treatment options, lifestyle modifications, including control diet and physical exercises, is proposed in order to obtain weight loss [5557]. It was estimated that each kilogram of fat mass lost is associated with simultaneously increase in plasma adiponectin level by approximately 6\% [58]. What is more, researches have shown that these methods also decrease insulin resistance, improve endothelial function, and reduce cardiovascular risk. Recent studies displayed that the most effective drugs with proven effects on bioavailability and concentrations of adiponectin are insulin sensitizers thiazolidinediones, peroxisome proliferator-activated receptor gamma (PPAR- $\gamma$ ) agonists, such as rosiglitazone, pioglitazone, hypoglycemic drugs as glimepiryd, cardiovascular medicament, like angiotensin receptor type 1 blockers (ARB family, mainly telmisartan, to a lesser degree candesartan and losartan), rennin-angiotensin system inhibitors (ACEI family, like temocapril, ramipril), and nebivolol, novel agent in beta-blocker group. It is also worth mentioning that both statins (simvastatin, atorvastatin, and rosuvastatin), diuretics, old betablockers, and metformin, the best known anti-hyperglycemic drugs, do not alter plasma adiponectin levels in obese patients with type 2 diabetes mellitus [28,53,54]. Moreover, the special attention is also paid to herbal medicines, such as garlic extract, astragaloside II and isoastragaloside I, isolated from medicina herb radix, Zataria multiflora plant, commonly known as Avishan-E-Shirazi, as these natural substances are suggested to play a role in 
increasing adiponectin levels, and at the same time alleviating insulin resistance and modulating vasculature function [53]. There are also some evidence that omega-3 fatty acids, cobalt, L-cysteine, and manganese supplementation can be supportive therapeutic strategy, although further studies are necessary [53,59]. The analysis of existing data indicates that roux-en-y gastric bypass surgery should be considered, especially in patients with severe obesity, as the treatment method allowing to not only weight loss, but also inducing sustained increases in plasma adiponectin levels with simultaneously improvement of the metabolic profile that is observed post-surgically [60-62]. It is also worth nothing that short-term weight loss do not change adiponectin concentration and insulin resistance, but only significant increment of HMW and reduction of MMW form.

Increased serum or plasma adiponectin levels after different treatment interventions prove its regulatory role and properties to improve and mimic metabolic and vascular actions of insulin and its protective influence on endothelial function in experimental and clinical studies. Constant development of various strategies involving both regulation of adiponectin and its receptors expression as well as discovering adiponectin receptors' agonists may make adiponectin as a multipotent therapeutic object to combat with obesity, insulin resistance, and their metabolic and vascular complications.

\section{Conclusions}

To conclude, it is worth to emphasize that obesity is a chronic fatal disease in which subclinical inflammatory process together with hipoadiponectinemia lead to disruption of vascular homeostasis and endothelial dysfunction, and these changes contribute to significantly higher risk of adverse cardiovascular events as a consequence. Adiponectin, an adipose tissue-derived hormone with insulin-sensitizing, anti-atherogenic, and antiinflammatory properties, may be a predictive factor of endothelial dysfunctionality and vascular remodeling development in the group of patients with overweight, obesity, and insulin resistance. Discovering of pharmacological agents and non-pharmacological interventions that increase the level of circulating adiponectin, would become novel and innovative therapeutic strategy to ameliorate obesity-related comorbidities. Therefore, further studies are required to determine the effect of adiponectin and its role in the pathogenesis metabolic diseases.

\section{References}

1. Engin A. The Definition and Prevalence of Obesity and Metabolic Syndrome. Adv Exp Med Biol. 2017;960:1-17. doi:10.1007/978-3-319-48382-5_1. 
2. Owens TM. Morbid obesity: the disease and comorbidities. Crit Care Nurs Q. 2003;26(2):162-165. doi:10.1097/00002727-200304000-00011.

3. Elagizi A, Kachur S, Lavie CJ, Carbone S, Pandey A, Ortega FB, et al. An Overview and Update on Obesity and the Obesity Paradox in Cardiovascular Diseases. Prog Cardiovasc Dis. 2018;61(2):142-150. doi:10.1016/j.pcad.2018.07.003.

4. Kramer H, Cao G, Dugas L, Luke A, Cooper R, Durazo-Arvizu R. Increasing BMI and waist circumference and prevalence of obesity among adults with Type 2 diabetes: the National Health and Nutrition Examination Surveys. J Diabetes Complications. 2010;24(6):368-374. doi:10.1016/j.jdiacomp.2009.10.001.

5. Kelly T, Yang W, Chen CS, Reynolds K, He J. Global burden of obesity in 2005 and projections to 2030. Int J Obes (Lond). 2008;32(9):1431-1437. doi:10.1038/ijo.2008.102.

6. Andolfi C, Fisichella PM. Epidemiology of Obesity and Associated Comorbidities. J Laparoendosc Adv Surg Tech A. 2018;28(8):919-924. doi:10.1089/lap.2018.0380.

7. Prospective Studies Collaboration, Whitlock G, Lewington S, Sherliker P, Clarke R, Emberson J, Halsey J, et al. Body-mass index and cause-specific mortality in 900000 adults: collaborative analyses of 57 prospective studies. Lancet. 2009;373(9669):10831096. doi:10.1016/S0140-6736(09)60318-4.

8. Murphy N, Cross AJ, Abubakar M, Jenab M, Aleksandrova K, Boutron-Ruault MC, et al. A Nested Case-Control Study of Metabolically Defined Body Size Phenotypes and Risk of Colorectal Cancer in the European Prospective Investigation into Cancer and Nutrition (EPIC). PLoS Med. 2016;13(4):e1001988. doi:10.1371/journal.pmed.1001988.

9. Boeing H, Weisgerber UM, Jeckel A, Rose HJ, Kroke A. Association between glycated hemoglobin and diet and other lifestyle factors in a nondiabetic population: cross-sectional evaluation of data from the Potsdam cohort of the European Prospective Investigation into Cancer and Nutrition Study. Am J Clin Nutr. 2000;71(5):1115-1122. doi:10.1093/ajen/71.5.1115.

10. Lear SA, James PT, Ko GT, Kumanyika S. Appropriateness of waist circumference and waist-to-hip ratio cutoffs for different ethnic groups. Eur J Clin Nutr. 2010;64(1):42-61. doi:10.1038/ejen.2009.70.

11. Poulos SP, Hausman DB, Hausman GJ. The development and endocrine functions of adipose tissue. Mol Cell Endocrinol. 2010;323(1):20-34. doi:10.1016/j.mce.2009.12.011.

12. Smekal A, Vaclavik J. Adipokines and cardiovascular disease: A comprehensive review. Biomed Pap Med Fac Univ Palacky Olomouc Czech Repub. 2017;161(1):31-40. doi:10.5507/bp.2017.002. 
13. Unamuno $X$, Gómez-Ambrosi $J$, Rodríguez A, Becerril S, Frühbeck G, Catalán V. Adipokine dysregulation and adipose tissue inflammation in human obesity. Eur J Clin Invest. 2018;48(9):e12997. doi:10.1111/eci.12997.

14. Landecho MF, Tuero C, Valentí V, Bilbao I, de la Higuera M, Frühbeck G. Relevance of Leptin and Other Adipokines in Obesity-Associated Cardiovascular Risk. Nutrients. 2019;11(11):2664. doi:10.3390/nu11112664.

15. Shehzad A, Iqbal W, Shehzad O, Lee YS. Adiponectin: regulation of its production and its role in human diseases. Hormones (Athens). 2012;11(1):8-20. doi:10.1007/BF03401534.

16. Wong GW, Krawczyk SA, Kitidis-Mitrokostas C, Revett T, Gimeno R, Lodish HF. Molecular, biochemical and functional characterizations of $\mathrm{C} 1 \mathrm{q} / \mathrm{TNF}$ family members: adipose-tissue-selective expression patterns, regulation by PPAR-gamma agonist, cysteine-mediated oligomerizations, combinatorial associations and metabolic functions. Biochem J. 2008;416(2):161-177. doi:10.1042/BJ20081240.

17. Ortiz Segura MDC, Del Río Navarro BE, Rodríguez Espino BA, Marchat LA, Sánchez Muñoz F, Villafaña S, et al. Abnormality of adipokines and endothelial dysfunction in Mexican obese adolescents with insulin resistance. Endocr Res. 2017;42(3):252-259. doi:10.1080/07435800.2017.1294601.

18. Liberale L, Bonaventura A, Vecchiè A, Casula M, Dallegri F, Montecucco F, et al. The Role of Adipocytokines in Coronary Atherosclerosis [published correction appears in Curr Atheroscler Rep. 2017 May;19(5):21]. Curr Atheroscler Rep. 2017;19(2):10. doi:10.1007/s11883-017-0644-3.

19. Nakamura K, Fuster JJ, Walsh K. Adipokines: a link between obesity and cardiovascular disease. J Cardiol. 2014;63(4):250-259. doi:10.1016/j.jjcc.2013.11.006.

20. Adeva-Andany MM, Martínez-Rodríguez J, González-Lucán M, Fernández-Fernández C, Castro-Quintela E. Insulin resistance is a cardiovascular risk factor in humans. Diabetes Metab Syndr. 2019;13(2):1449-1455. doi:10.1016/j.dsx.2019.02.023.

21. Rohde K, Keller M, la Cour Poulsen L, Blüher M, Kovacs P, Böttcher Y. Genetics and epigenetics in obesity. Metabolism. 2019;92:37-50. doi:10.1016/j.metabol.2018.10.007.

22. Saltiel AR, Olefsky JM. Inflammatory mechanisms linking obesity and metabolic disease. J Clin Invest. 2017;127(1):1-4. doi:10.1172/JCI92035.

23. Murano I, Barbatelli G, Parisani V, Latini C, Muzzonigro G, Castellucci M, et al. Dead adipocytes, detected as crown-like structures, are prevalent in visceral fat depots of 
genetically obese mice. J Lipid Res. 2008;49(7):1562-1568. doi:10.1194/jlr.M800019JLR200.

24. Sun K, Tordjman J, Clément K, Scherer PE. Fibrosis and adipose tissue dysfunction. Cell Metab. 2013;18(4):470-477. doi:10.1016/j.cmet.2013.06.016.

25. Crewe C, An YA, Scherer PE. The ominous triad of adipose tissue dysfunction: inflammation, fibrosis, and impaired angiogenesis. J Clin Invest. 2017;127(1):74-82. doi:10.1172/JCI88883.

26. Rodríguez A, Ezquerro S, Méndez-Giménez L, Becerril S, Frühbeck G. Revisiting the adipocyte: a model for integration of cytokine signaling in the regulation of energy metabolism. Am J Physiol Endocrinol Metab. 2015;309(8):E691-E714. doi:10.1152/ajpendo.00297.2015.

27. Kwaifa IK, Bahari H, Yong YK, Noor SM. Endothelial Dysfunction in Obesity-Induced Inflammation: Molecular Mechanisms and Clinical Implications. Biomolecules. 2020;10(2):291. doi:10.3390/biom10020291.

28. Balsan GA, Vieira JL, Oliveira AM, Portal VL. Relationship between adiponectin, obesity and insulin resistance. Rev Assoc Med Bras (1992). 2015;61(1):72-80. doi:10.1590/1806-9282.61.01.072.

29. Smitka K, Marešová D. Adipose Tissue as an Endocrine Organ: An Update on Proinflammatory and Anti-inflammatory Microenvironment. Prague Med Rep. 2015;116(2):87-111. doi:10.14712/23362936.2015.49.

30. Nakano Y, Tobe T, Choi-Miura NH, Mazda T, Tomita M. Isolation and characterization of GBP28, a novel gelatin-binding protein purified from human plasma. J Biochem. 1996;120(4):803-812. doi:10.1093/oxfordjournals.jbchem.a021483.

31. Metin Aksu N, Yazgan Aksoy D, Akkaş M, Çinar N, Uçar F, Bülent Yildiz O, et al. Adiponectin levels decrease independently of body mass index and diabetes type after the normalization of hyperglycemia. Turk J Med Sci. 2020;50(2):312-315. doi:10.3906/sag1903-35.

32. Engin A. Adiponectin-Resistance in Obesity. Adv Exp Med Biol. 2017;960:415-441. doi:10.1007/978-3-319-48382-5_18.

33. Kocełak P, Chudek J, Olszanecka-Glinianowicz M. Prevalence of metabolic syndrome and insulin resistance in overweight and obese women according to the different diagnostic criteria. Minerva Endocrinol. 2012;37(3):247-254. 
34. Caceres M, Teran CG, Rodriguez S, Medina M. Prevalence of insulin resistance and its association with metabolic syndrome criteria among Bolivian children and adolescents with obesity. BMC Pediatr. 2008;8:31. doi:10.1186/1471-2431-8-31.

35. Bacha F, Saad R, Gungor N, Arslanian SA. Adiponectin in youth: relationship to visceral adiposity, insulin sensitivity, and beta-cell function. Diabetes Care. 2004;27(2):547-552. doi:10.2337/diacare.27.2.547.

36. Ayina CN, Noubiap JJ, Etoundi Ngoa LS, Boudou F, Gautier JF, Mengnjo MK, et al. Association of serum leptin and adiponectin with anthropomorphic indices of obesity, blood lipids and insulin resistance in a Sub-Saharan African population. Lipids Health Dis. 2016;15:96. doi:10.1186/s12944-016-0264-x.

37. Han SJ, Boyko EJ, Fujimoto WY, Kahn SE, Leonetti DL. Low Plasma Adiponectin Concentrations Predict Increases in Visceral Adiposity and Insulin Resistance. J Clin Endocrinol Metab. 2017;102(12):4626-4633. doi:10.1210/jc.2017-01703.

38. Hara K, Horikoshi M, Yamauchi T, et al. Measurement of the high-molecular weight form of adiponectin in plasma is useful for the prediction of insulin resistance and metabolic syndrome. Diabetes Care. 2006;29(6):1357-1362. doi:10.2337/dc05-1801.

39. Liu W, Zhou X, Li Y, Zhang S, Cai X, Zhang R, et al. Serum leptin, resistin, and adiponectin levels in obese and non-obese patients with newly diagnosed type 2 diabetes mellitus: A population-based study. Medicine (Baltimore). 2020;99(6):e19052. doi:10.1097/MD.0000000000019052.

40. Kuo SM, Halpern MM. Lack of association between body mass index and plasma adiponectin levels in healthy adults. Int J Obes (Lond). 2011;35(12):1487-1494. doi:10.1038/ijo.2011.20.

41. Mongraw-Chaffin M, Foster MC, Anderson CAM, Kalyani RR, Vaidya D, Burke GL, et al. Metabolically Healthy Obesity, Transition to Metabolic Syndrome, and Cardiovascular Risk. J Am Coll Cardiol. 2018;71(17):1857-1865. doi:10.1016/j.jacc.2018.02.055.

42. Smith GI, Mittendorfer B, Klein S. Metabolically healthy obesity: facts and fantasies. J Clin Invest. 2019;129(10):3978-3989. doi:10.1172/JCI129186.

43. Stefan N, Häring HU, Schulze MB. Metabolically healthy obesity: the low-hanging fruit in obesity treatment?. Lancet Diabetes Endocrinol. 2018;6(3):249-258. doi:10.1016/S2213-8587(17)30292-9. 
44. Eckel N, Meidtner K, Kalle-Uhlmann T, Stefan N, Schulze MB. Metabolically healthy obesity and cardiovascular events: A systematic review and meta-analysis. Eur J Prev Cardiol. 2016;23(9):956-966. doi:10.1177/2047487315623884.

45. Engin A. Endothelial Dysfunction in Obesity. Adv Exp Med Biol. 2017;960:345-379. doi:10.1007/978-3-319-48382-5_15.

46. Muñoz-Muñoz E, Krause BJ, Uauy R, Casanello P. LGA-newborn from patients with pregestational obesity present reduced adiponectin-mediated vascular relaxation and endothelial dysfunction in fetoplacental arteries. J Cell Physiol. 2018;233(10):6723-6733. doi:10.1002/jcp.26499.

47. Satish M, Saxena SK, Agrawal DK. Adipokine Dysregulation and Insulin Resistance with Atherosclerotic Vascular Disease: Metabolic Syndrome or Independent Sequelae?. J Cardiovasc Transl Res. 2019;12(5):415-424. doi:10.1007/s12265-019-09879-0.

48. Oishi JC, Castro CA, Silva KA, Fabricio V, Cárnio EC, Phillips SA, et al. Endothelial Dysfunction and Inflammation Precedes Elevations in Blood Pressure Induced by a HighFat Diet [published correction appears in Arq Bras Cardiol. 2019 Jan;112(1):116]. Arq Bras Cardiol. 2018;110(6):558-567. doi:10.5935/abc.20180086.

49. Ohashi K, Kihara S, Ouchi N, Kumada M, Fujita K, Hiuge A, et al. Adiponectin replenishment ameliorates obesity-related hypertension [published correction appears in Hypertension. 2007;49(2):e14]. Hypertension. 2006;47(6):1108-1116. doi:10.1161/01.HYP.0000222368.43759.a1.

50. Li R, Wang WQ, Zhang H, Yang X, Fan Q, Christopher TA, et al. Adiponectin improves endothelial function in hyperlipidemic rats by reducing oxidative/nitrative stress and differential regulation of eNOS/iNOS activity. Am J Physiol Endocrinol Metab. 2007;293(6):E1703-E1708. doi:10.1152/ajpendo.00462.2007.

51. Matsuda M, Shimomura I, Sata M, Arita Y, Nishida M, Maeda M, et al. Role of adiponectin in preventing vascular stenosis. The missing link of adipo-vascular axis. $\mathrm{J}$ Biol Chem. 2002;277(40):37487-37491. doi:10.1074/jbc.M206083200.

52. Beatty AL, Zhang $\mathrm{MH}, \mathrm{Ku} I A, \mathrm{Na} B$, Schiller NB, Whooley MA. Adiponectin is associated with increased mortality and heart failure in patients with stable ischemic heart disease: data from the Heart and Soul Study. Atherosclerosis. 2012;220(2):587-592. doi:10.1016/j.atherosclerosis.2011.11.038.

53. Achari AE, Jain SK. Adiponectin, a Therapeutic Target for Obesity, Diabetes, and Endothelial Dysfunction. Int J Mol Sci. 2017;18(6):1321. doi:10.3390/ijms18061321. 
54. Han SH, Quon MJ, Kim JA, Koh KK. Adiponectin and cardiovascular disease: response to therapeutic interventions. J Am Coll Cardiol. 2007;49(5):531-538. doi:10.1016/j.jacc.2006.08.061.

55. Korybalska K, Luczak J, Swora-Cwynar E, Kanikowska A, Czepulis N, Kanikowska D, et al. Weight loss-dependent and -independent effects of moderate calorie restriction on endothelial cell markers in obesity. J Physiol Pharmacol. 2017;68(4):597-608.

56. Rocha-Rodrigues S, Gonçalves IO, Beleza J, Ascensão A, Magalhães J. Physical exercise mitigates high-fat diet-induced adiposopathy and related endocrine alterations in an animal model of obesity. J Physiol Biochem. 2018;74(2):235-246. doi:10.1007/s13105018-0609-1.

57. Corbi G, Polito R, Monaco ML, Cacciatore F, Scioli M, Ferrara N, et al. Adiponectin Expression and Genotypes in Italian People with Severe Obesity Undergone a Hypocaloric Diet and Physical Exercise Program. Nutrients. 2019;11(9):2195. doi:10.3390/nu11092195.

58. Calvani M, Scarfone A, Granato L, Mora EV, Nanni G, Castagneto M, et al. Restoration of adiponectin pulsatility in severely obese subjects after weight loss. Diabetes. 2004;53(4):939-947. doi:10.2337/diabetes.53.4.939.

59. Huang F, Del-Río-Navarro BE, Leija-Martinez J, Torres-Alcantara S, Ruiz-Bedolla E, Hernández-Cadena L, et al. Effect of omega-3 fatty acids supplementation combined with lifestyle intervention on adipokines and biomarkers of endothelial dysfunction in obese adolescents with hypertriglyceridemia. J Nutr Biochem. 2019;64:162-169. doi:10.1016/j.jnutbio.2018.10.012.

60. Chai F, Wang Y, Zhou Y, Liu Y, Geng D, Liu J. Adiponectin downregulates hyperglycemia and reduces pancreatic islet apoptosis after roux-en-y gastric bypass surgery. Obes Surg. 2011;21(6):768-773. doi:10.1007/s11695-011-0357-6.

61. Wolf RM, Jaffe AE, Steele KE, Schweitzer MA, Magnuson TH, Wolfe A, et al. Cytokine, Chemokine, and Cytokine Receptor Changes Are Associated With Metabolic Improvements After Bariatric Surgery. J Clin Endocrinol Metab. 2019;104(3):947-956. doi:10.1210/jc.2018-02245.

62. Farias G, Netto BDM, Boritza K, Bettini SC, Vilela RM, Dâmaso AR. Impact of Weight Loss on Inflammation State and Endothelial Markers Among Individuals with Extreme Obesity After Gastric Bypass Surgery: a 2-Year Follow-up Study. Obes Surg. 2020;30(5):1881-1890. doi:10.1007/s11695-020-04411-9. 